\title{
Emulsion Microgel Particles as High-Performance Bio-Lubricants
}

\author{
Ophelie Torres, Efren Andablo-Reyes, Brent S. Murray, and Anwesha Sarkar*(1) \\ Food Colloids and Processing Group, School of Food Science and Nutrition, University of Leeds, Leeds, LS2 9JT, U.K.
}

\section{Supporting Information}

\begin{abstract}
Starch-based emulsion microgel particles with different starch (15 and $20 \mathrm{wt} \%)$ and oil contents (0-15 wt \%) were synthesized, and their lubrication performance under physiological conditions was investigated. Emulsion microgels were subjected to skin mimicking or oral cavity mimicking conditions, i.e., smooth hydrophobic polydimethylsiloxane ball-ondisc tribological tests, in the absence or presence of salivary enzyme ( $\alpha$-amylase). In the absence of enzyme, emulsion microgel particles (30-60 vol \% particle content) conserved the lubricating properties of emulsion droplets, providing consid-

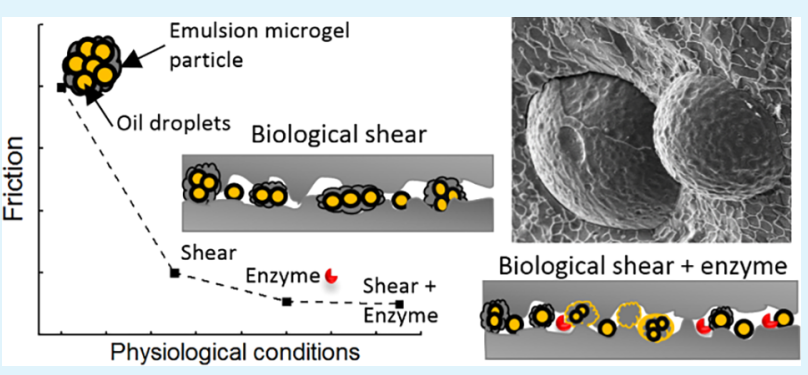
erably lower friction coefficients $(\mu \leq 0.1)$ in the mixed lubrication regime compared to plain microgel particles ( 0 wt \% oil). Upon addition of enzyme, the lubrication performance of emulsion microgel particles became strongly dependent on the particles' oil content. Microgel particles encapsulating 5-10 wt \% oil showed a double plateau mixed lubrication regime having a lowest friction coefficient $\mu \sim 0.03$ and highest $\mu \sim 0.1$, the latter higher than with plain microgel particles. An oil content of $15 \mathrm{wt} \%$ was necessary for the microgel particles to lubricate similarly to the emulsion droplets, where both systems showed a normal mixed lubrication regime with $\mu \leq 0.03$. The observed trends in tribology, theoretical considerations, and the combined results of rheology, light scattering, and confocal fluorescence microscopy suggested that the mechanism behind the low friction coefficients was a synergistic enzyme- and shear-triggered release of the emulsion droplets, improving lubrication. The present work thus demonstrates experimentally and theoretically a novel biolubricant additive with stimuli-responsive properties capable of providing efficient boundary lubrication between soft polymeric surfaces. At the same time, the additive should provide an effective delivery vehicle for oil soluble ingredients in aqueous media. These findings demonstrate that emulsion microgel particles can be developed into multifunctional biolubricant additives for future use in numerous soft matter applications where both lubrication and controlled release of bioactives are essential.
\end{abstract}

KEYWORDS: microgel, lubricant additives, soft tribology, friction reduction, biolubricant, encapsulation, colloidal stability, biological enzymes

\section{INTRODUCTION}

Biolubricants are in great demand to reduce friction between soft biological contacting surfaces, such as the eyes, the oral cavity, the gastrointestinal tract, and joints, to prevent discomfort and wear of epithelial tissues and cartilage. ${ }^{1-5}$ The use of submicron to micron sized particles (e.g., microgels) as biolubricant additives has recently been a focal point in biomaterial science research due to their ability to reduce friction and wear in the boundary regime of sliding contact in biological areas, such as tongue-oral palate contacts, cartilage, or eye blinking. ${ }^{1,2,6,7}$ At low sliding speeds and contact pressures, these particles are able to enter the gap and reduce direct contact between the biological surfaces by acting as "true surface separators" by virtue of their rheological properties. $^{8}$ The extent to which the friction reduces can be controlled by the particle properties, such as their size, volume fraction, surface roughness, and mechanical response.

Oil-in-water emulsions are widely used as lubricants in pharmaceutical, personal care, and food products. The lubrication properties of emulsions primarily arise from the oil droplets in the boundary regime, which is observed at the lowest entrainment speed where the friction force is independent of the entrainment speed and the load is supported predominantly by the asperity contact and surface adsorbed matter. ${ }^{7,9}$ While emulsions demonstrate lubrication properties in both external (in the form of topical creams to create a protective barrier increasing the moisture content or allow the triggered release of a lipophilic bioactive under mechanical shear ${ }^{10}$ ) and internal applications (in the form of medicine or food to coat the oral cavity, reduce friction and increase palatability ${ }^{11,12}$ ), their instability to physicochemical and biochemical degradation (e.g., light, temperature, shear, ionic and enzymatic activity) can lead to poor release of bioactive compounds and increased physiological oxidation of the oil droplets. ${ }^{13-15}$ Therefore, being able to tailor microstructures that combine high lubrication performances, protection of colloidal droplets against such physicochemical degradation, and controlled release of bioactive compounds under either enzymatic- or mechanical shear-trigger is highly desirable. Nevertheless, most targeted release studies of

Received: May 14, 2018

Accepted: July 23, 2018

Published: July 23, 2018 
bioactive material do not consider the effect of degradation caused by biological shear or enzymatic stresses. Emulsion microgel particles are a new class of microgel particle where several oil droplets are trapped within a biopolymer hydrogel particle. ${ }^{16-18}$ In this study we demonstrate for the first time how, by employing suitable novel biocompatible emulsion microgel particles, it is possible to provide protection to emulsion droplets combined with the desired lubrication under relevant physiological shear and/or enzymatic conditions. By engineering the appropriate biopolymer matrix, enzymetriggered and/or mechanical shear-triggered release of the emulsion droplets can also be observed. To demonstrate the applied physiological performance of these emulsion microgel particles, we subjected them to both external and internal environmental stress models (i.e., tribological shear and enzymatic stress, respectively). A series of starch based emulsion microgel particles were produced at different starch (15 and 20 wt \%) and sunflower oil (0-15 wt \%) concentrations via a top down approach. ${ }^{18}$ Starch was employed due to its known response to $\alpha$-amylase in the oral cavity. The lubrication performance of the emulsion microgel particles was examined in the absence or presence of $\alpha$-amylase using hydrophobic polydimethylsiloxane (PDMS) tribo-pairs (water contact angle $108^{\circ}$ ), due to their resemblance to soft biological interfaces., ${ }^{79}$ For a better understanding of the lubrication mechanisms of the different samples, the theoretical drag force as well as the indentation of the different particles were calculated. The viscosity, particle size, and microstructure of the microgel dispersions at various length scales were investigated before and after tribological shear in the absence or presence of $\alpha$-amylase.

The results demonstrated that in conventional oil-in-water emulsions the emulsion droplets coalesced releasing a thin oil film under shear and/or enzyme activity providing good lubrication properties. In comparison, the emulsion microgel particles under tribological shear and enzyme activity led to the release of very few emulsion droplets improving lubrication without coalescence of the oil droplets. The noticeable stability of emulsion microgel particles offers immense potential for targeted release of emulsion droplets containing bioactive molecules at physiological sites (typically the small intestine for internal applications or the epidermis for cutaneous applications) while reducing friction in aqueous biological environments that has wide potential soft matter industrial and biological applications in food, pharmaceutical, and personal care industries. For instance in the case of cutaneous applications, emulsion microgel particles offer a promising silky lubrication effect without causing a greasy sticky layer at the epidermis due to the coalescence of emulsion droplets and the formation of an oil film under mechanical shear. In the case of oral applications, emulsion microgel particles containing active compounds (such as nutraceuticals or pharmaceutics) should provide the desirable creamy palatable mouthfeel $^{11}$ without the destabilization of the emulsion droplets which occurs in a conventional emulsion. ${ }^{20,21}$ This protection might further allow for the bioactive molecules to be released at targeted physiological sites. ${ }^{1,10,22-26}$

\section{EXPERIMENTAL SECTION}

2.1. Materials. Wheat native starch was purchased from SigmaAldrich (Gillingham, U.K.), commercial octenyl succinic anhydride (OSA) starch refined from waxy maize starch from Ingredion (Hamburg, Germany) was used as an emulsifier, and sunflower oil was obtained from Morrisons (U.K.) supermarket. $\alpha$-Amylase from porcine pancreas Type VI-B (13 units $\mathrm{mg}^{-1}$ ) was obtained from Sigma-Aldrich and dissolved in $\mathrm{pH} 6.8$ phosphate buffered saliva when used. All other chemicals were of Analytical grade and purchased from Sigma-Aldrich unless otherwise specified. All dispersions were prepared with Milli- $Q$ water having a resistivity of not less than $18.2 \mathrm{M} \Omega \mathrm{cm}$ at $25{ }^{\circ} \mathrm{C}$ (Milli-Q apparatus, Millipore, Bedford, U.K.).

2.2. Preparation of Starch Based Emulsion Microgel Particles. Starch emulsion microgel particles at different concentrations of starch (15-20 wt \%) and oil-in-water emulsions (0-15 wt $\%)$ were prepared using a top-down approach, as reported previously. ${ }^{18}$ First, 40 wt $\%$ sunflower oil was emulsified with 4 wt $\%$ OSA starch. Second, starch pastes were formed by dispersing native wheat starch into Milli- $\mathrm{Q}$ water, heating at $80{ }^{\circ} \mathrm{C}$ for $40 \mathrm{~min}$ in a water bath, and simultaneously sheared using a hand blender (Hand blender, XB986B, 170W, Argos, U.K.). Both starch pastes and emulsions were then mixed at different ratios to form emulsion gels. After cooling to $4{ }^{\circ} \mathrm{C}$ for $3 \mathrm{~h}$, the refrigerated starch and starch emulsion gels were passed twice through a high-pressure homogenizer at 250/50 bar (Panda Plus, GEA Niro Soave, Parma, Italy). The resulting particles were collected in a beaker and immediately diluted in Milli-Q water (60:40 w/w, particles, Milli-Q).

2.3. Preparation of Model Saliva ( $\mathrm{pH}$ 6.8). Phosphate buffer containing $75 \mathrm{U} \mathrm{mL}^{-1} \alpha$-amylase was prepared according to the composition used in previous literature, ${ }^{27}$ mimicking the ionic composition and $\mathrm{pH}$ of saliva. The different samples were mixed gently with buffer (with or without $\alpha$-amylase) in $1: 1 \mathrm{w} / \mathrm{w}$ ratio based on the oral processing protocol of the standardized static in vitro digestion method. ${ }^{27}$

2.4. Particle Size Analysis. The particle size distributions of the emulsion droplets and emulsion microgel particles were measured via a Malvern Mastersizer 3000E Hydro, (Malvern Instruments, Worcestershire, U.K.) using refractive indices of the emulsion droplets, starch microgel particles, and aqueous phase of 1.46, 1.50, and 1.33 , respectively.

2.5. Shear Rheology. Rheological characterization was performed using a Kinexus ultra rheometer (Malvern Instruments Ltd., Worcestershire, U.K.) with a cone and plate geometry (diameter 40 $\mathrm{mm}$, model CP4/40 SS017SS). In order to prevent sample evaporation, in addition to the use of an adiabatic cover, the rim of the geometry was sealed with a thin layer of $350 \mathrm{cst}$ silicone oil. Flow curves were obtained from the different samples before and after dilution (1:1) with buffer (with and without $\alpha$-amylase) as a function of shear rate, ranging from 0.01 to $1000 \mathrm{~s}^{-1}$ at $37^{\circ} \mathrm{C}$.

2.6. Tribology. The lubrication performance of the emulsions, starch microgel particles (i.e., without oil droplets), and starch emulsion microgel particles was assessed using a Mini Traction Machine (MTM2, PCS Instruments, U.K.) following the methods outlined in previous studies. ${ }^{7,28}$ The tribo-pairs used for this study consisted of a ball (diameter $19 \mathrm{~mm}$ ) and plate (diameter $46 \mathrm{~mm}$ ) made of hydrophobic polydimethylsiloxane (PDMS, Sylgard, Down Corning), each element having a surface roughness of $\left(R_{\mathrm{a}}\right)<50 \mathrm{~nm}$ and Young's modulus of $2.8 \mathrm{MPa}$. All tests were performed at constant normal load $\left(W_{\mathrm{T}}\right)$ of $2 \mathrm{~N}$, equivalent to a maximum Hertzian contact pressure $\left(P_{\max }\right)$ of $100 \mathrm{kPa}$ and contact radius of $2 \mathrm{~mm}$. The friction coefficient $(\mu)$, defined as the ratio between the friction force and normal load, was measured as a function of entrainment speed $(\bar{U})$ swept between 1 and $500 \mathrm{~mm} \mathrm{~s}^{-1}$ in both descending and ascending directions. Only data obtained from entrainment speed sweeps descending from 500 to $1 \mathrm{~mm} \mathrm{~s}^{-1}$ are reported, as the Stribeck curves showed negligible hysteresis. The sliding to rolling ratio, defined as $S R R=\left|U^{\prime}-U^{\prime \prime}\right| \mid \bar{U}$ was kept at a constant value of 0.5 , where $U^{\prime}$ and $U^{\prime \prime}$ are the ball and disc speeds, respectively, and the entrainment speed is defined as $\bar{U}=\frac{1}{2}\left(U^{\prime}+U^{\prime \prime}\right)$. Prior to each test, the surfaces were cleaned with acetone and rinsed with Milli- $Q$ water. One ball-and-disk pair was used each time for an individual experiment and then discarded. Tests were performed under isothermal conditions at $37 \pm 1{ }^{\circ} \mathrm{C}$, aiming to mimic human oral 
(a)

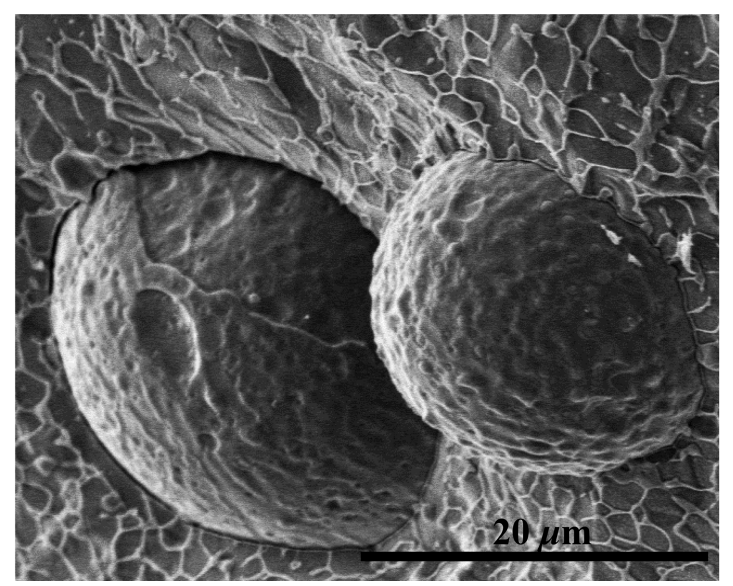

(b)

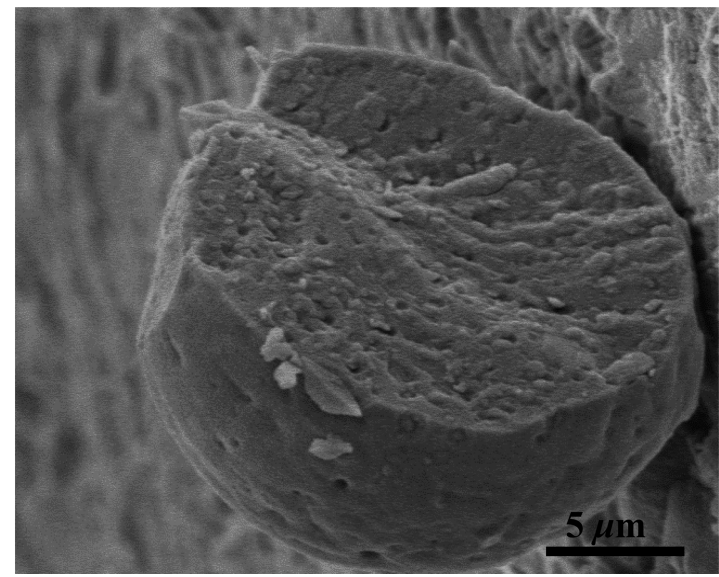

Figure 1. (a) Cryo-SEM micrograph of the external structure and (b) internal structure of starch emulsion microgel particles (15 wt $\%$ starch-10 wt $\%$ oil), respectively. Scale bar represents $5 \mu \mathrm{m}$.
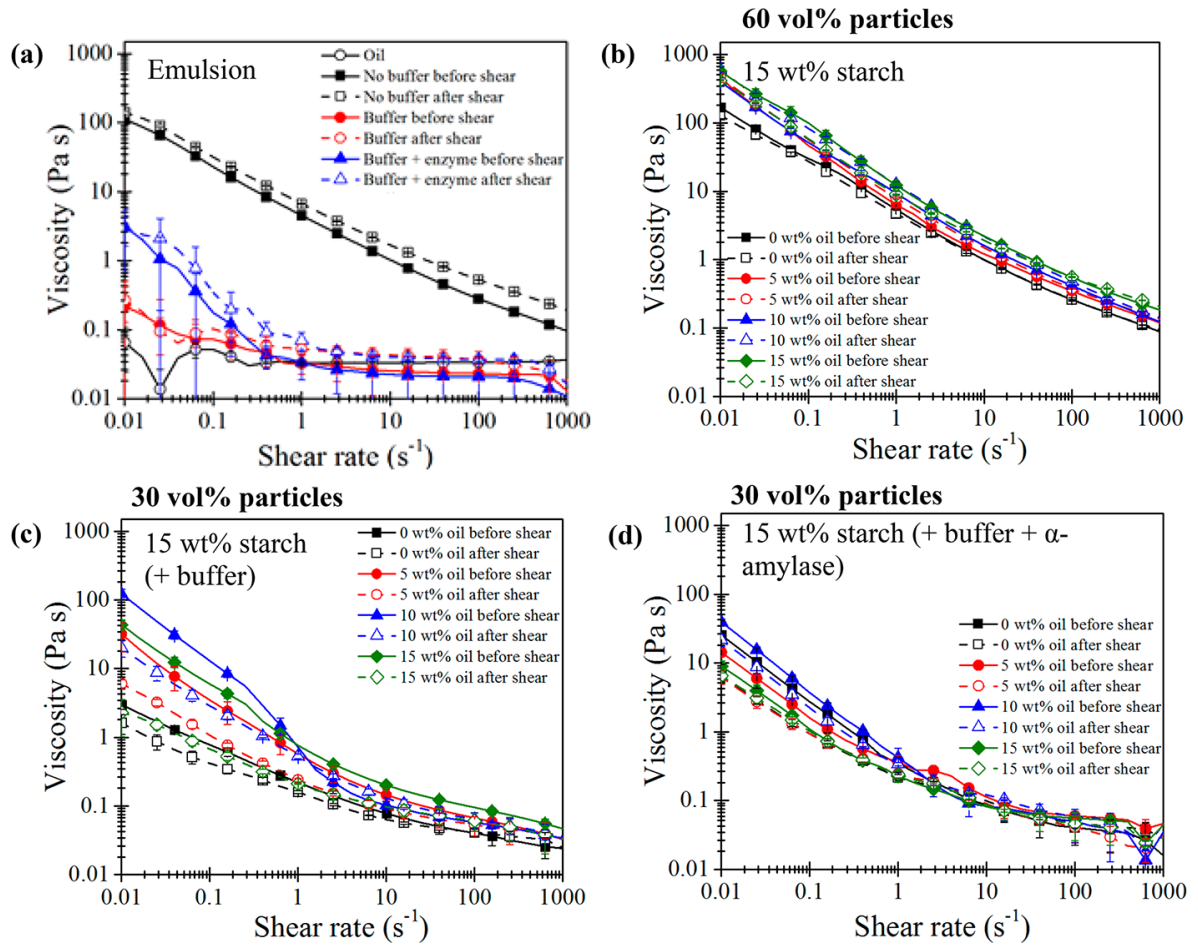

Figure 2. (a) Viscosity versus shear rate of the oil-in-water emulsion at $37^{\circ} \mathrm{C}$ before and after tribological shear in the absence or presence of buffer and $\alpha$-amylase, viscosity versus shear rate of the emulsion microgel particles $(15 \mathrm{wt} \% \mathrm{starch})$ at different concentrations of oil at $37^{\circ} \mathrm{C}$, before and after tribological shear in the absence of buffer (b), in the presence of buffer (c), and $\alpha$-amylase (d), respectively.

conditions. The mean value of three measurements for each sample was used to plot the Stribeck curve.

2.7. Confocal Scanning Laser Microscopy (CLSM). The microstructure of the OSA starch-stabilized emulsion and the emulsion microgel particles ( $15 \mathrm{wt} \%$ starch $-15 \mathrm{wt} \%$ oil) at time 0 and $60 \mathrm{~s}$ after incorporating buffer with $\alpha$-amylase (at $37^{\circ} \mathrm{C}$ ) was studied via a Zeiss LSM 880 confocal microscope (Carl Zeiss MicroImaging GmbH, Jena, Germany), at a $40 \times$ magnification. The samples were stained following the methods from previous studies. ${ }^{18}$

2.8. Cryo-Scanning Electron Microscopy (cryo-SEM). A cryoscanning electron microscope (FEI Quanta 200F FEG ESEM, Japan), equipped with a Quorum PolarPrep 2000 cryo-system was used to study the structural features of the emulsion microgel particles following the methods used in previous studies. ${ }^{17,18}$

\section{RESULTS AND DISCUSSION}

3.1. Morphological Characterization of Emulsion Microgel Particles by cryo-SEM. Cryo-scanning electron micrographs of the emulsion microgel particles at various magnifications are presented in Figure 1. The particles appeared to be roughly spherical with an average diameter of $20 \mu \mathrm{m}$. Their rough surface has been previously assumed to be the underlying intact encapsulated oil droplets, ${ }^{18}$ which might impact the tribological performance of the particles. Under higher magnification, the internal structure of a fragmented particle can be observed (Figure $1 \mathrm{~b}$ ), where spherical particles of $0.1-1 \mu \mathrm{m}$ can be seen. This size range is consistent with the size distribution of OSA starch-stabilized emulsion, which had an average Sauter mean diameter $d_{32}$ of $0.16 \pm 0.004 \mu \mathrm{m}$, similar to the value obtained in previous studies. ${ }^{18,29,30}$ 

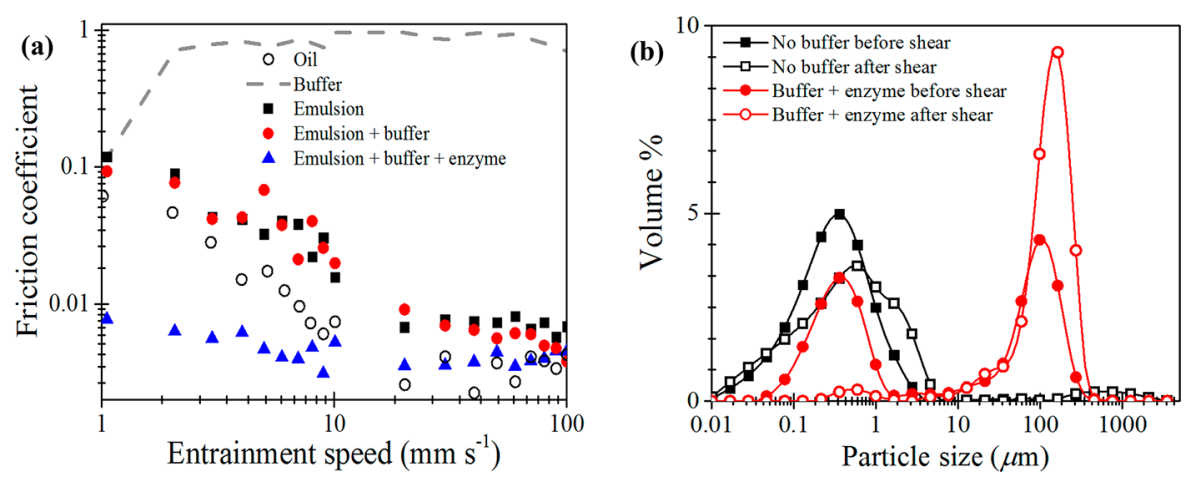

Figure 3. (a) Coefficient of friction as a function of entrainment speed for sunflower oil, buffer with $\alpha$-amylase and OSA starch stabilized emulsion in absence or presence of buffer and/or $\alpha$-amylase subjected to a normal load of $2 \mathrm{~N}$ and at $37^{\circ} \mathrm{C}$; (b) Particle size distribution of the OSA starch stabilized-emulsion ( $40 \mathrm{wt} \%$ oil) with and without $\alpha$-amylase before and after being subjected to tribological shear.

3.2. Rheological Characterization. The viscosities of the emulsion and emulsion microgel particles before and after tribological shear were measured to check how shear affected the samples. The Newtonian curve of sunflower oil with a viscosity of approximately $0.04 \mathrm{~Pa} \mathrm{~s}$ was introduced for comparison. The viscosity of the emulsion in the absence of buffer and $\alpha$-amylase decreased with increasing shear rate (Figure 2a), i.e., the emulsion exhibited typical shear thinning of a weakly flocculated system. ${ }^{31}$ Viscosity measurements of the emulsion after tribological shear (without buffer or $\alpha$ amylase) indicated, on average, a $50 \%$ increase in viscosity over the shear rate range, suggesting an increase in droplet aggregation. Depletion flocculation due to nonabsorbed OSA starch possibly contributed to this increased droplet aggregation. $^{32}$

The 1:1 dilution of the emulsion with buffer (without $\alpha$ amylase) led to a 100 -fold decrease in viscosity, over the same shear rate range showing further evidence of weak flocculation at the higher volume fraction (40 wt \% oil) but largely absent at 20 wt $\%$ oil. Additionally, for the latter, a high shear rate viscosity plateau of approximately $0.04 \mathrm{~Pa}$ s can be observed at shear rates $>1 \mathrm{~s}^{-1}$, where shear-induced forces reach a balance and the average interparticle structures are highly reduced in extent or completely absent.

Emulsions in the presence of buffer and $\alpha$-amylase showed a more pronounced shear thinning behavior compared to emulsions diluted only with buffer. This is consistent with flocculation due to decreased steric stabilization induced by the addition of $\alpha$-amylase to the OSA starch-stabilized emulsion, which is expected to hydrolyze the OSA starch chains adsorbed at the oil droplet surface. ${ }^{32-34}$ The high shear rate viscosity plateau is observed to start approximately at the same shear rate and have a similar value to the emulsion without $\alpha$ amylase, showing that both systems have a very similar state of dispersion in this limit, probably with a few particle clusters surviving. Regarding the emulsion microgel particles, only the viscosity measurements obtained with 15 wt \% starch particles at different oil concentrations are shown in Figure $2 b-d$. The measurements obtained at $20 \mathrm{wt} \%$ starch are reported in the Supporting Information (Figure S1) since they followed very similar trends. However, emulsion microgel particles produced at $20 \mathrm{wt} \%$ starch had in average a viscosity $50 \%$ higher than the particles produced at $15 \mathrm{wt} \%$ starch.

In Figure $2 b$, it is seen that emulsion microgel particle suspensions exhibited very similar flow curves in shape and absolute values of viscosity compared to nondiluted emulsions, despite the higher solids content, approximately double the former. This might be related to the relatively low elastic modulus of microgel particles which has been reported to have a large effect in the rheology of this type of system. ${ }^{35}$ This assumption is strengthened by the influence of increasing oil content of the emulsion microgel particles, which significantly increased the viscosity values. For instance, at $40 \mathrm{~s}^{-1}$ the viscosities of the emulsion microgel particles produced with 0 , 5,10 , and 15 wt $\%$ oil were, $0.42,0.56,0.70$, and $0.94 \pm 0.05$ $\mathrm{Pa} \mathrm{s}$, respectively. Thus, encapsulating over 5 wt \% oil significantly increased the viscosity of the starch particle dispersions $(p<0.05)$. It is also seen that tribological shear did not significantly affect the rheological properties of the suspensions (Figure 2b). The dilution of the emulsion microgel particles in buffer $(50: 50 \mathrm{w} / \mathrm{w})$ did not affect the shear thinning behavior of the samples (Figure 2c), but the viscosity of all samples decreased on average by 1 order of magnitude, in line with previous studies on the effect of microgel particle volume fraction on viscosity. ${ }^{7,36}$

A nonmonotonic relationship between shear viscosity and emulsion microgel particle oil content can also be observed due to the relatively broader size distribution of microgel emulsions with $15 \%$ oil content compared to systems with lower oil concentrations. At lower volume fractions, particles are well separated, allowing the continuous phase to flow in between them. ${ }^{37}$ The tribological shear applied to the emulsion microgel particles further decreased the viscosity of all the samples, suggesting that the shear further separated or damaged the aggregated emulsion microgel particles.

The addition of $\alpha$-amylase to the emulsion microgel particles did not affect their shear thinning behavior; their viscosity values were similar to the emulsion microgel particles diluted with buffer without $\alpha$-amylase (Figure 2d). Interestingly, however, in contrast to the systems diluted with buffer, when $\alpha$-amylase was present as well, tribological shear seemed to have less effect in reducing the viscosities, so that all the flow curves before and after shear were closer (Figure 2d). The reasons for this are not clear, but the rheology of partly enzyme degraded microgel particles and the starch fragments released, is not expected to be simple. Nevertheless, it is necessary to make these measurements to aid interpretation of the subsequent studies described in the next section.

3.3. Tribological Properties of the Emulsion. Figure 3a shows the friction coefficient of the OSA-stabilized emulsion under different conditions as a function of entrainment speed. 
(a)

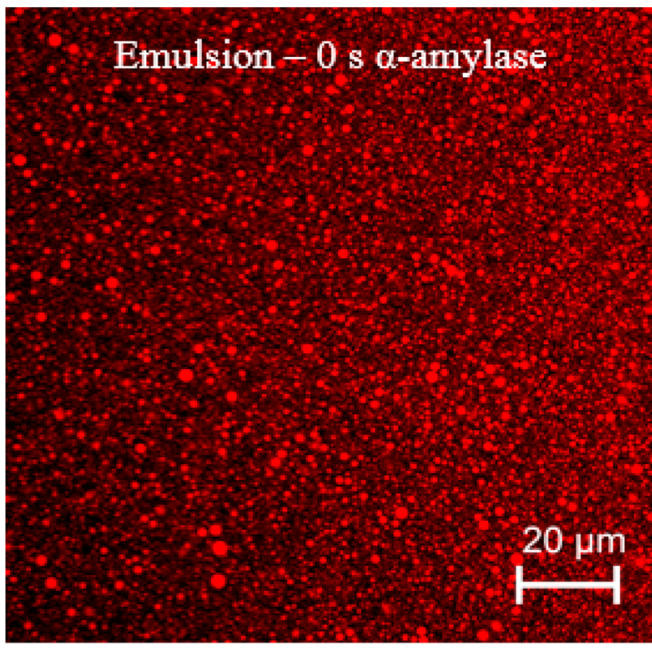

(c)

Emulsion

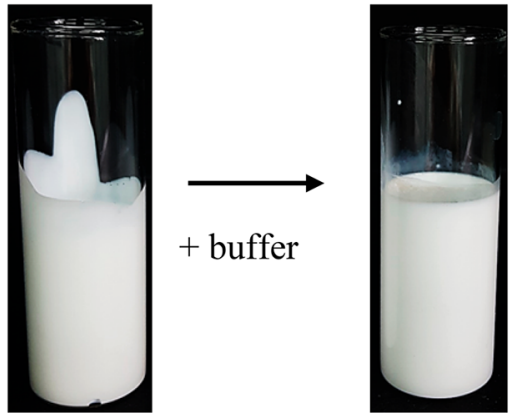

(b)

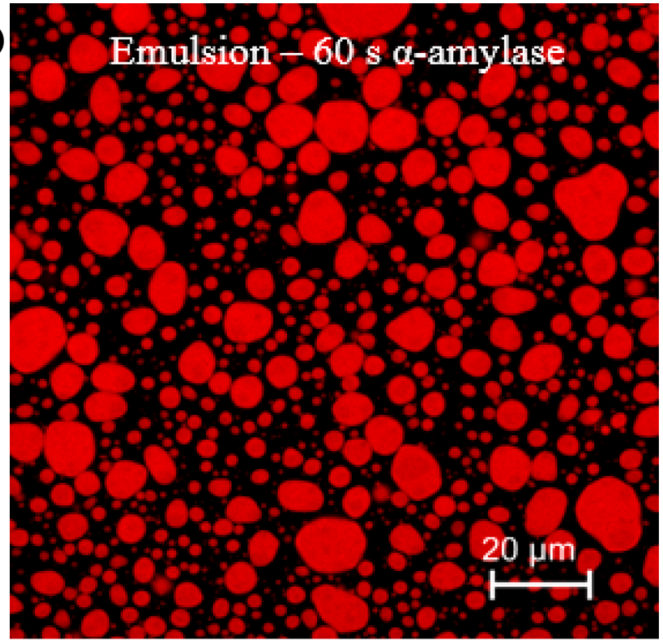

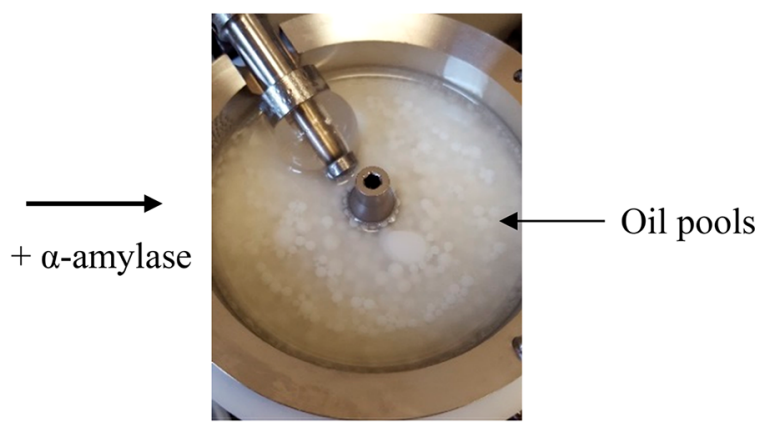

Figure 4. Confocal fluorescence images of the emulsion $0 \mathrm{~s}$ (a) and $60 \mathrm{~s}$ (b) after the addition of $\alpha$-amylase before tribological shear $(\lambda=488 \mathrm{~nm}$, oil droplets); (c) photographs of the emulsions in the absence or presence of buffer and/or $\alpha$-amylase in the tribometer.

Sunflower oil and buffer without $\alpha$-amylase were also measured for comparison purposes.

Previously, it has been reported that under normal circumstances three tribological regimes can be observed. ${ }^{7,12,38}$ At low entrainment speed, the PDMS ball and disc are in contact with each other, excluding the sample from the contact area, resulting in a high friction coefficient. This condition is known as the boundary regime. As the entrainment speed increases, sample is entrained in between the ball and disc, forming a monolayer that decreases the friction: the mixed regime (the amount of entrainment will mainly depend on the size of the particles as compared to the size of the gap between the ball and disc). As the speed increases further, the amount of sample entering the contact area increases, pushing the ball and disc further apart reducing the friction further until the hydrodynamic regime is reached, where bulk properties rather than surface properties dominate. Since this study aims to understand biological surfaces relevant to oral or dermal applications, only the boundary and mixed regime will be discussed in detail. However, data in the hydrodynamic regime can be found in the Supporting Information (Figure S2).

In Figure $3 \mathrm{a}$, the contacts lubricated with buffer worked only in the boundary lubrication regime, with high friction coefficient values $\mu \sim 0.7$ for the whole range of entrainment speeds, showing practically no penetration of the lubricant into the contact area. Significantly different behavior was displayed when lubricated with emulsions containing $40 \mathrm{wt} \%$ (no added buffer) and 20 wt $\%$ oil (with added buffer). The contacts exhibited the characteristics of working in the mixed lubrication regime with values of friction coefficient remarkably lower compared to buffer (see the Supporting Information,
Table S3 for statistical analysis) and decreasing from 0.1 to approximately 0.007 with increasing entrainment speed. Comparing the Stribeck curves obtained for emulsions and sunflower oil, both systems provided very similar lubricating properties (Table S3). This has been observed in previous studies, where the dispersed phase of an emulsion had a viscosity at least four times larger than the that of the continuous phase, ${ }^{39}$ similar to the case of the systems studied here. However, the emulsion without added buffer (40 wt \% oil) plateaued at a low friction coefficient $\left(\bar{U}>50 \mathrm{~mm} \mathrm{~s}^{-1}, \mu=\right.$ $0.007 \pm 0.004)$ whereas the emulsion diluted with buffer $(20$ wt \% oil) clearly reached its elastohydrodynamic regime $(\bar{U}>$ $100 \mathrm{~mm} \mathrm{~s}^{-1}$ ) (Figure S2). At high entrainment speeds, the lubrication mechanism of the emulsion diluted with buffer might be controlled by sunflower oil, as compared to the emulsion without buffer, which might be controlled by the emulsion droplets. These different mechanisms will be discussed in more detail further on.

In parallel, the droplet size distribution of the undiluted emulsion before and after tribological shear was also examined (Figure $3 \mathrm{~b}$ ). The oil droplet size distribution before shear was monomodal and narrow, with a mean droplet size $d_{32}=0.16 \pm$ $0.004 \mu \mathrm{m}$. After shearing, the size distribution of the oil droplets became wider and more skewed toward higher diameters values, although the mean droplet size remained at $d_{32}=0.16 \pm 0.021 \mu \mathrm{m}$. Under tribological shear, the oil droplets might be pressed together to enter the thin contact zone, leading to minor oil droplet aggregation. ${ }^{40}$ To recall, an increase in viscosity of the emulsion was observed, which can be related to such shear induced aggregation (Figure $2 \mathrm{a}$ ). The addition of buffer $+\alpha$-amylase to the emulsion decreased the 

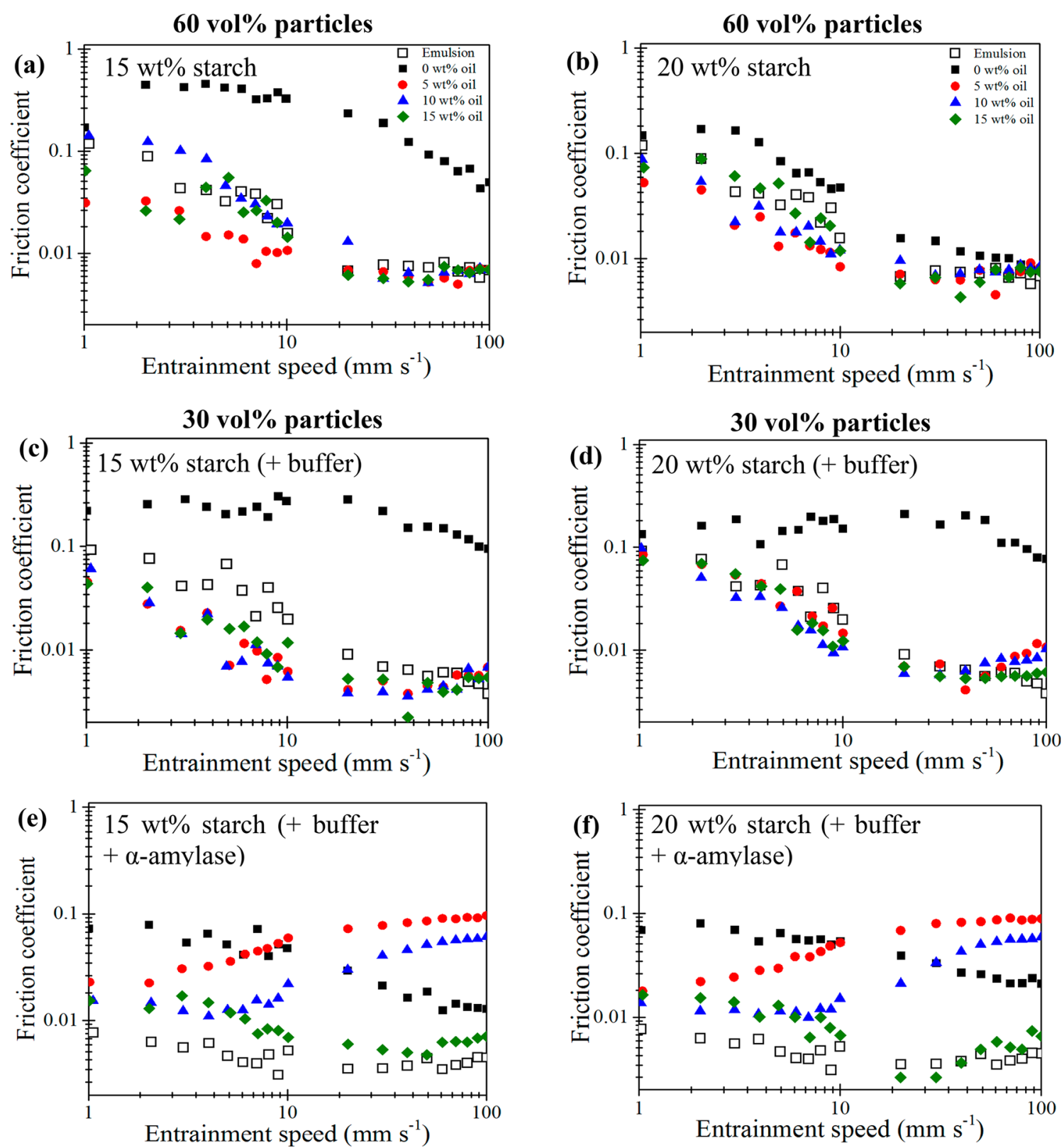

Figure 5. Coefficient of friction as a function of entrainment speed of starch microgel particles encapsulating different oil content measured at $2 \mathrm{~N}$ and $37{ }^{\circ} \mathrm{C}$ in absence of buffer and $\alpha$-amylase $(\mathrm{a}, \mathrm{b})$; in the presence of buffer $(50: 50 \mathrm{w} / \mathrm{w})$ without $\alpha$-amylase $(\mathrm{c}, \mathrm{d})$; in the presence of buffer $(50: 50 \mathrm{w} / \mathrm{w})$ with $\alpha$-amylase $(\mathrm{e}, \mathrm{f})$. Controls are the OSA-stabilized emulsion under the same conditions.

friction coefficient values compared to sunflower oil, suggesting the destabilization of the emulsions. OSA starch adsorbed at the oil-water interface might be expected to be hydrolyzed, leading to oil droplet destabilization, as described above in the rheology section.

In addition, the oil droplet size distribution became bimodal on addition of $\alpha$-amylase before being subjected to tribological shear (Figure $3 b$ ). Interestingly, the oil droplet size distribution with $\alpha$-amylase reverted to a monomodal distribution at the higher end of the particle size range (i.e., $100 \mu \mathrm{m}$ ), after shearing. This drastic increase in size also suggested that the tribological shear enhanced the destabilization and coalescence of the emulsions in contact with $\alpha$-amylase, at least for the smaller emulsion droplets, confirmed by visual observations as shown in Figure 4c.
The microstructure of the OSA starch-stabilized emulsions at 0 and $60 \mathrm{~s}$ after adding $\alpha$-amylase (at $37{ }^{\circ} \mathrm{C}$ ) was investigated in situ via confocal fluorescence microscopy (Figure 4a,). Without enzymes, the oil droplets were uniformly distributed (Figure 4a). After $60 \mathrm{~s}$ with enzyme, much larger oil droplets were observable and the system was polydispersed (Figure $4 \mathrm{~b}$ ), in agreement with the light scattering results. These results are also in accordance with previous studies. ${ }^{34}$ In summary, it seems possible to mimic the lubrication properties of sunflower oil using oil-in-water emulsions when subjecting them to oral processing conditions relevant to the operating conditions in the mouth.

3.4. Tribological Properties of the Emulsion Microgel Particles. To recapitulate, in spite of the good lubrication properties of a 40 wt \% oil-in-water emulsion, due to the oil droplet coalescence induced by the shear $+\alpha$-amylase activity, 

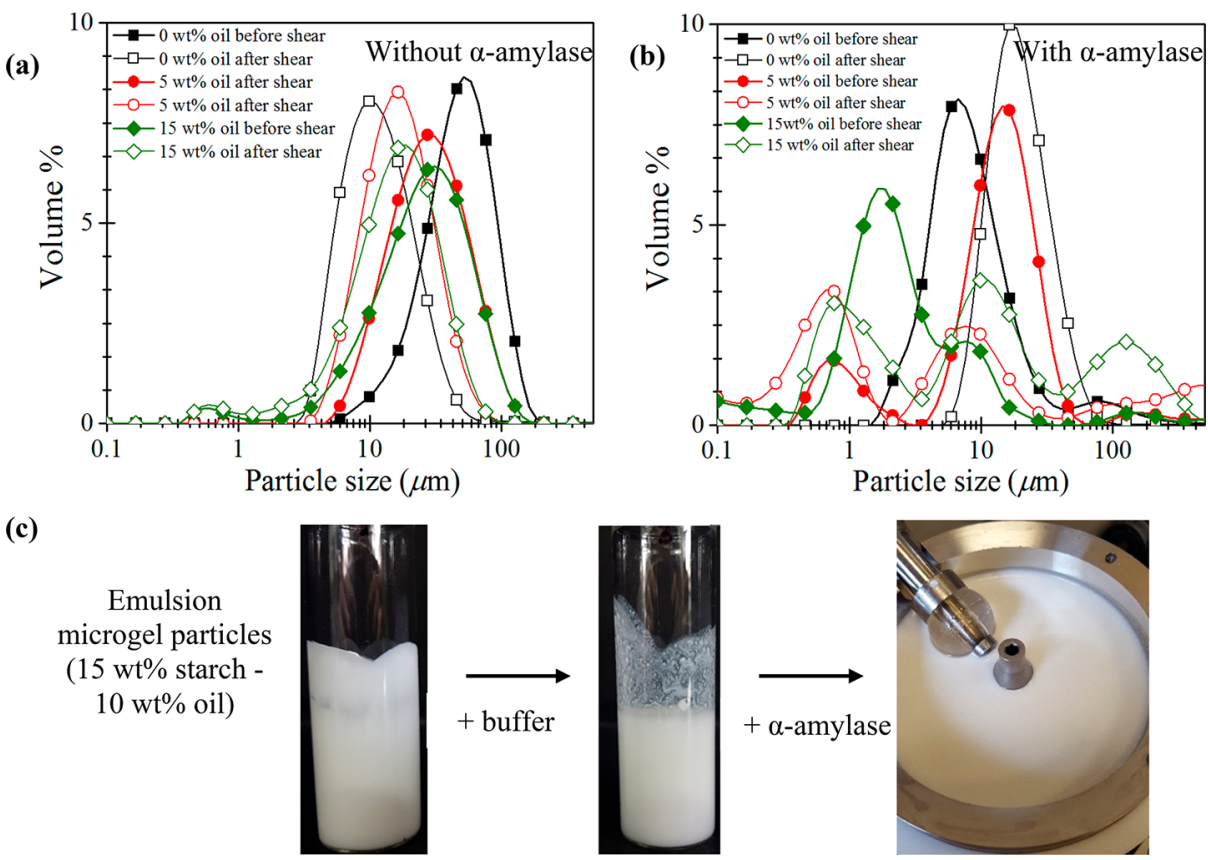

Figure 6. Particle size distribution of $15 \mathrm{wt} \%$ starch particles encapsulating different oil concentrations before or after being sheared by the tribometer being in the absence (a) or presence of $\alpha$-amylase (b) and (c) photographs of the emulsions after tribology in the absence or presence of buffer and/or $\alpha$-amylase.

this poor emulsion stability and therefore poor controlled release would be of great concern in many applications, such as liquid medicines. To overcome this drawback, the encapsulation of the emulsion droplets into starch microgel particles might allow better stability and controlled release than the emulsion itself, without compromising lubrication even at much lower oil content.

The tribological properties of the starch emulsion microgel particles (15 and $20 \mathrm{wt} \%$ starch; 0-15 wt \% oil) were investigated for $1<\bar{U}<100 \mathrm{~mm} \mathrm{~s}^{-1}$ under the same conditions as above (Figure 5). Results at $\bar{U}>100 \mathrm{~mm} \mathrm{~s}^{-1}$ can be found in the Supporting Information Figure S4. Corresponding Stribeck curves of the emulsion, previously shown in Figure 3, are also shown in Figure 5 in order to aid comparison.

Starch microgel particles without oil droplets (15 and $20 \mathrm{wt}$ $\%$ starch-0 wt \% oil), displayed significant differences in friction coefficient and in the limit of their boundary region (Figure 5a,b and Supporting Information Table S7). The $15 \mathrm{wt}$ $\%$ starch particles had a friction coefficient of $0.460 \pm 0.068$ at $\bar{U}=3 \mathrm{~mm} \mathrm{~s}^{-1}$ and $0.093 \pm 0.014$ at $\bar{U}=50 \mathrm{~mm} \mathrm{~s}^{-1}$ and entered the mixed regime $>10 \mathrm{~mm} \mathrm{~s}^{-1}$. In comparison, $20 \mathrm{wt} \%$ starch particles had a friction coefficient of $0.166 \pm 0.028$ at $\bar{U}$ $=3 \mathrm{~mm} \mathrm{~s}^{-1}$ and $0.011 \pm 0.002$ at $\bar{U}=50 \mathrm{~mm} \mathrm{~s}^{-1}$ and entered the mixed regime $<5 \mathrm{~mm} \mathrm{~s}^{-1}$. Assuming that the starch microgel particles have the same elastic modulus as the starch gel, the particles would have an elastic modulus of $G^{\prime}=640$ $\mathrm{Pa}^{18}$ and behave as flexible and deformable soft solids. Therefore, at low entrainment speed $\left(\bar{U} \leq 10 \mathrm{~mm} \mathrm{~s}^{-1}\right), 15$ wt $\%$ starch particles might enter the thin ball and disc gap and flatten out ${ }^{41}$ (Figure 5a). Such particles would not be capable of supporting the load, which would increase the contact area causing an increase in the friction coefficient. This effect has previously been described as resulting from a sliding motion of the sample, retarding the onset of the mixed regime. ${ }^{41}$ Increasing the starch concentration to $20 \mathrm{wt} \%$ resulted in harder and less deformable particles $\left(G^{\prime}=1600 \mathrm{~Pa}^{18}\right)$, which would only enter the contact zone when the gap between the ball and disc reached the size of the particles. ${ }^{42}$ As soon as such particles entered the contact zone, these harder particles would be able to act as surface separators by carrying some part of the load, fast-tracking the onset of the mixed regime. ${ }^{43}$ Of course the difference in $G^{\prime}$ between the two particle types is less than a factor of 3, but any other explanation alludes us at present.

The encapsulation of oil droplets $(5-15 \mathrm{wt} \%$ oil) into the starch particles led to similar friction coefficient values as compared to the emulsion (without buffer). The encapsulation of a small amount of oil thus seemed to allow the starch particles to have emulsion-like friction properties with negligible influence of the starch particles themselves. Similar results have previously been reported with the use of fluid gels. ${ }^{19}$ However, increasing the oil content further did not seem to significantly influence the friction coefficient values (see Supporting Information Tables S5 and S6 for statistical analysis). The 1:1 dilution of the particles by buffer mainly influenced the friction properties of the starch particles without oil droplets, whereas the friction measurements of all the emulsion microgel particles did not seem to be affected by this dilution (Figure 5c,d and Tables S7 and S8). Both types of microgel particles behaved in a similar manner, with high friction $(\mu>0.2)$ in the boundary regime $\left(\bar{U} \leq 50 \mathrm{~mm} \mathrm{~s}^{-1}\right)$ and a decreased friction in the mixed regime $(50 \leq \bar{U} \leq 100$ $\mathrm{mm} \mathrm{s}^{-1}$ ). Previous studies have demonstrated that the volume fraction of particles influences the friction coefficient in the boundary regime. ${ }^{7,9}$ A low volume fraction of particles increases the friction coefficient, since less particles can be entrained into the contact zone, as compared to a high volume fraction of particles.

The addition of $\alpha$-amylase drastically affected the friction properties of the different samples (Figure 5e,f). Both starch particles without oil droplets (15 and $20 \mathrm{wt} \%$ starch) only displayed a mixed regime throughout the entrainment speed ( 1 
(a)

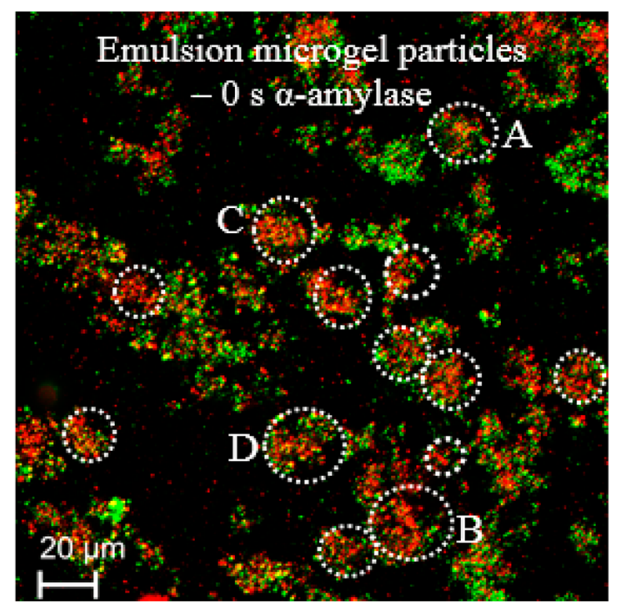

(b)

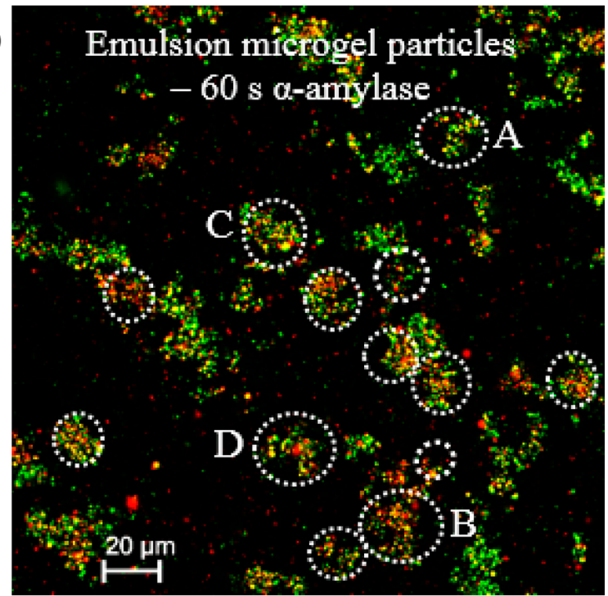

Figure 7. Confocal fluorescence images of the emulsion microgel particles $0 \mathrm{~s}$ (a) and $60 \mathrm{~s}$ (b) after the addition of $\alpha$-amylase $(\lambda=488 \mathrm{~nm}$, oil droplets and $639 \mathrm{~nm}$, starch shell), scale bar represents $20 \mu \mathrm{m}$.

$\left.\leq \bar{U} \leq 100 \mathrm{~mm} \mathrm{~s}^{-1}\right)$. A significant reduction in friction coefficient compared to that with the diluted starch particles without $\alpha$-amylase was also noticeable above $\bar{U} \geq 50 \mathrm{~mm} \mathrm{~s}^{-1}$ (see the Supporting Information Table S8 for statistical analysis).

For example, at $\bar{U}=50 \mathrm{~mm} \mathrm{~s}^{-1}, 15$ wt \% starch particles in buffer without $\alpha$-amylase $\mu=0.156 \pm 0.015$, whereas, with $\alpha$ amylase $\mu=0.019 \pm 0.006$. This reduction in the friction coefficient might be explained by the degradation of the starch particles via the $\alpha$-amylase into smaller microgel particles $\left(d_{32}\right.$ $=18.5 \pm 1.6 \mu \mathrm{m})$ as compared to the initial starch particles $\left(d_{32}=39.9 \pm 1.1 \mu \mathrm{m}\right)$ (Figure 6). Smaller particles can obviously more easily enter the tribo-pairs gap, separating the ball and disc at a lower entrainment speed and hence reducing the friction coefficient at a lower speed. ${ }^{19,31}$

In the case of the emulsion microgel particles in the presence of $\alpha$-amylase, at low entrainment speed $\left(1 \leq \bar{U} \leq 10 \mathrm{~mm} \mathrm{~s}^{-1}\right)$, their friction coefficient decreased below the values of the emulsion in buffer but were still above the value for the emulsion with $\alpha$-amylase (compare Figure 5e,f to Figure 3a). For example at $\bar{U}=3 \mathrm{~mm} \mathrm{~s}^{-1}$, the emulsion had a friction coefficient of $\mu=0.0056 \pm 0.001$ and 15 wt $\%$ starch with $5 \mathrm{wt}$ $\%$ oil $\mu=0.016 \pm 0.008$. The higher friction coefficient of the emulsion microgel particles as compared to the emulsion with $\alpha$-amylase suggested that the $\alpha$-amylase started digesting the starch particle shell, allowing some stable oil droplets to be released in the continuous phase. Some degraded starch particles might also aid the reduction in friction by reducing the contact area further. However, any oil droplets released from the emulsion microgel particles did not appear to coalesce during the tribology measurements, no free oil could be observed (Figure 6c). This is very different from the emulsion on its own, where phase separation was evident (Figure 4). Possibly the $\alpha$-amylase activity was saturated by the starch shell, so that the OSA starch adsorbed at the oil interface was not significantly digested.

Additionally, it is worth noting that in the presence of enzymes, the oil concentration did seem to affect the friction values. For example, at $\bar{U}=50 \mathrm{~mm} \mathrm{~s}^{-1}$ the friction coefficient of $20 \mathrm{wt} \%$ starch particle with 5, 10, and $15 \mathrm{wt} \%$ oil were $0.083 \pm 0.003,0.050 \pm 0.001$, and $0.005 \pm 0.001$, respectively (see Supporting Information Table S8 for statistical analysis). By increasing the entrainment speed $\left(\bar{U} \geq 10 \mathrm{~mm} \mathrm{~s}^{-1}\right)$, the friction values of the emulsion microgel particles produced at 5 and 10 wt $\%$ oil increased, which might indicate jamming of the samples around the ball and disc contact zone. ${ }^{42,44}$ Since the emulsion microgel particles do slightly break down under the effect of shear and enzyme hydrolysis (see below), some empty and/or smaller starch emulsion microgel particles might aggregate and build-up around the contact zone. This buildup of aggregated particles would prevent the entrainment of the sample, ${ }^{3}$ increasing the friction, until the entrainment speed reached $100 \mathrm{~mm} \mathrm{~s}^{-1}$. In comparison, the emulsion microgel particles with $15 \mathrm{wt} \%$ oil showed a decrease in friction between 10 to $100 \mathrm{~mm} \mathrm{~s}^{-1}$. However, as the entrainment speed increased further $\left(\bar{U} \geq 100 \mathrm{~mm} \mathrm{~s}^{-1}\right)$, the elastohydrodynamic regime was reached (Figure S3).The higher filler to matrix ratio might have produced a thinner and more brittle starch shell around the oil droplets, allowing for a higher oil content to be released between the gap, under tribological shear. Under the mixed regime this would have prevented the jamming of the particles at the contact zone, reducing the friction coefficient. Further evidence for the explanation given above was obtained by measuring the particle size distribution (PSD) of the systems before and after tribological shear, combined with microscopic imaging described below.

3.5. Particle Size Distribution (PSD) before and after Tribological Shear. The PSDs of 15 and $20 \mathrm{wt} \%$ starch microgel particles had the same size, hence only the results obtained for $15 \mathrm{wt} \%$ starch particles are presented in Figure 6 (the results obtained for $20 \mathrm{wt} \%$ starch particles can be found in the Supporting Information (Figure S9)). Additionally, because essentially the same behavior was observed for $15 \mathrm{wt}$ $\%$ starch emulsion microgel particles with 5, 10, and 15 wt \% oil encapsulated, for clarity only results for 5 and $15 \mathrm{wt} \%$ oil are shown. In the absence of $\alpha$-amylase (Figure 6a) before tribological shear all samples had a monomodal particle size distribution, ranging in size between 10 and $100 \mu \mathrm{m}$. After tribological shear, the PSDs of all the samples were similar, although shifted to smaller sizes, between 5 and $60 \mu \mathrm{m}$. It is worth noting that the shift in PSD was greater for the starch microgel particles without oil droplets compared to those containing oil droplets. Possibly, this was due to the oil droplets strengthening the microgel particles as an active filler. ${ }^{18}$ The PSD changes also fit in with the proposed explanation of the viscosity changes described earlier, in terms of breakup of aggregates with increasing shear rate. 
Table 1. Relative Indentation and Drag Force Calculations of the Emulsion Droplets of Radius $0.08 \mu \mathrm{m}$ and Particles of Radius $15 \mu \mathrm{m}$ at $\bar{U}=3 \mathrm{~mm} \mathrm{~s}^{-1}$ and $\phi_{\mathrm{p}}=20 \%$

\begin{tabular}{|c|c|c|c|c|c|}
\hline lubricant type & $W_{\mathrm{L}}(\%)$ & $\delta \mathrm{R}^{*}$ & $\eta$ at $0.01 \mathrm{~s}^{-1}(\mathrm{~Pa} \mathrm{~s})$ & $W_{\mathrm{p}}(\mathrm{N})$ & $F_{\mathrm{d}}(\mathrm{N})$ \\
\hline emulsion (40 wt \% oil) & 86 & 0.72 & 100 & $1.3 \times 10^{-8}$ & $4.5 \times 10^{-6}$ \\
\hline emulsion + buffer $(20$ wt $\%$ oil $)$ & 86 & 0.72 & 0.1 & $1.3 \times 10^{-8}$ & $9.1 \times 10^{-9}$ \\
\hline 15 wt $\%$ starch particles (60 vol \%) & 29 & 18.7 & 100 & $1.5 \times 10^{-4}$ & $8.5 \times 10^{-4}$ \\
\hline 20 wt $\%$ starch particles ( 60 vol \%) & 71 & 18.7 & 1000 & $3.7 \times 10^{-4}$ & $8.5 \times 10^{-3}$ \\
\hline emulsion microgel particles (60 vol \%) & 86 & 12.7 & 500 & $4.5 \times 10^{-4}$ & $4.5 \times 10^{-3}$ \\
\hline emulsion microgel particles (30 vol \%) & 86 & 12.7 & 200 & $4.5 \times 10^{-4}$ & $1.7 \times 10^{-3}$ \\
\hline
\end{tabular}

In the presence of $\alpha$-amylase, Figure $6 \mathrm{~b}$ shows that the main effect of tribological shear was to produce a much broader PSD than in the absence of amylase for all the microgel particles. Thus, the enzyme activity seemed to enhance the production of both smaller fragments and larger clusters $(0.1-100 \mu \mathrm{m})$, in line with previous studies. ${ }^{33}$ However, there was no evidence of coalescence (Figure 6c) so that larger particles in the PSD are more likely to be aggregates of microgel materials than large oil droplets.

3.6. Morphology of the Emulsion and Emulsion Microgel Particles. CLSM observations on the emulsion microgel particles (15 wt \% starch-10 wt \% oil) illustrated in Figure 7, suggest that most oil droplets (red) were completely entrapped in the starch gel matrix (green) at time $0 \mathrm{~s}$. In Figure $7 \mathrm{a}$, dotted lines have been added surrounding typical microgel regions $(A-D)$ before the addition of enzyme. After $60 \mathrm{~s}$ with enzyme, the same regions became smaller and less red, i.e., lower in oil content, suggesting their degradation and release of oil droplets (Figure $7 \mathrm{~b}$ ). However, no coalescence of the oil droplets can be observed in Figure $7 \mathrm{a}, \mathrm{b}$, confirming the previous observations that emulsion stability was not compromised when these were embedded in the emulsion microgel particles (Figure 6).

3.7. Lubrication Mechanism of Emulsion Microgel Particles. In order to understand the physical properties of the lubricant partially separating the contact surfaces, a mechanical analysis of the emulsion droplets, starch, and emulsion microgel particles in the contact area was introduced.

From the Hertz theory ${ }^{45}$ of contact points, the radius of contact $\alpha_{\mathrm{H}}$ and the indentation of the contact $\delta$ can be obtained. To establish the relative indentation of emulsion droplets or particles, the normal load supported by the lubricant $\left(W_{\mathrm{L}}\right)$ and by an individual emulsion droplet or particle $\left(W_{\mathrm{p}}\right)$ as well as the reduced elastic modulus of contacts formed by the particles and the PDMS contact surfaces $\left(E^{*}\right)$, were analyzed (see Supporting Information Figure S5 and Figure $3 \mathrm{a}$ and Figure 5). The number of particles of radius $R$, forming a monolayer, inside the contact $\left(N_{\mathrm{p}}\right)$ with an effective fraction of particles $\left(\phi_{\mathrm{p}}\right)$ covering the contact area $\left(a_{\mathrm{TP}}\right)$ were also estimated (see Supporting Information Figure S5).

Hence, the relative indentation for a monolayer of particles of a Poisson ratio $v$, in the radius of contact $\left(a_{\mathrm{H}}\right)$ can be expressed as eq 1 :

$$
\frac{\delta}{R}=\left(\frac{a_{\mathrm{H}}}{R}\right)^{2}-\frac{4}{3 \pi\left(1-v^{2}\right)}\left(\frac{a_{\mathrm{H}}}{R}\right)^{3} f\left(\frac{a_{\mathrm{H}}}{R}\right)
$$

where the ratio $a_{\mathrm{H}} / R$ is independent of $R$ and relates the relative indentation to the fraction of surface covered by particles $\phi_{\mathrm{p}}$ (eq 2):

$$
\frac{a_{\mathrm{H}}}{R}=\left(\frac{3 W_{\mathrm{L}}}{4 \phi_{\mathrm{P}} E^{*} a_{\mathrm{TP}}^{2}}\right)^{1 / 3}
$$

In order to understand if such emulsion droplets could be dragged into the contact zone the entrainment force on the particles at the vicinity of the contact was also estimated by using the Stokes drag eq 3:

$$
F_{\mathrm{d}}=6 \pi R \eta \bar{U}
$$

where, $\eta$ and $\bar{U}$ are the bulk viscosity and the entrainment speed, respectively. For particles to be entrained into the contact zone, the drag force $\left(F_{\mathrm{d}}\right)$ should be larger than the load applied to one particle $\left(W_{\mathrm{p}}\right)$.

Based on the experimental and theoretical results, we have summarized the relative indentation and drag force of the emulsion, emulsion in buffer, 60 vol \% starch particles produced at 15 and $20 \mathrm{wt} \%$ starch, 60 and $30 \mathrm{vol} \%$ emulsion microgel particles produced with $20 \mathrm{wt} \%$ starch and $10 \mathrm{wt} \%$ oil in Table 1. Due to the similarities of the other lubricants, their relative indentations and drag forces are not displayed. For both emulsions (before and after dilution in buffer), at an entrainment speed of $3 \mathrm{~mm} \mathrm{~s}^{-1}$ (Figure 3a), 86\% of the load was calculated to be supported by the emulsion droplets, with $\mu=0.1$ and $\mu_{\mathrm{B}}=0.7$. Therefore, at a Hertz contact radius of $2.07 \mathrm{~mm}$, for $20 \%$ fraction of emulsion droplets covering the contact surface $\left(\phi_{\mathrm{p}}\right)$ and $W_{\mathrm{p}}$ estimated at $1.3 \times 10^{-8} \mathrm{~N}$, the relative indentation of the emulsion droplets was calculated as 0.72 (Table 1). This implies that, on entering the ball and disc contact zone, an emulsion droplet of radius $0.08 \mu \mathrm{m}$ would compress to an elliptical shape of a height of $\sim 0.05 \mu \mathrm{m}$. The surface roughness of PDMS being $\left(R_{\mathrm{a}}\right) \leq 50 \mathrm{~nm}$, the compressed emulsion droplets might also enter the asperities of PDMS while still supporting $86 \%$ of the load resulting in the decrease in friction coefficient. However, due to the viscosity difference between the emulsion and the emulsion + buffer, the drag force necessary to entrain the emulsion droplets differed.

Before dilution, the high viscosity of the emulsion at low shear $(\eta>100 \mathrm{~Pa} \mathrm{~s})$ led to a large drag force $\left(F_{\mathrm{d}}>W_{\mathrm{p}}\right)$ as compared to the load applied to each emulsion droplets. The emulsion droplets would be entrained in between the tribo-pair via a sliding mechanism. The dilution of the emulsion with buffer reduced the viscosity $(\eta=0.2 \mathrm{~Pa} \mathrm{~s})$ of the emulsion as well as the volume fraction of oil entrained in between the tribo-pairs.

Although the friction coefficient obtained for the diluted emulsion did not differ from the initial emulsion at $\bar{U}=3 \mathrm{~mm}$ $\mathrm{s}^{-1}$, the low viscosity of the emulsion in buffer leads to a lower drag force with $F_{\mathrm{d}}<W_{\mathrm{p}}$, predicting emulsion droplets of 0.08 $\mu \mathrm{m}$ radius would not be entrained (Table 1 ). However, smaller emulsion droplets are also present that might be entrained and aggregate there, as suggested by the light scattering results 
Scheme 1. Schematic Representation of OSA Starch Stabilized Oil-in-Water Emulsion in the Mixed Regime of a Tribometer in the Absence (a) or Presence of Buffer (b) with and without $\alpha$-Amylase (c)

(a)

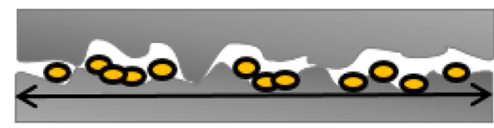

Contact area (b)

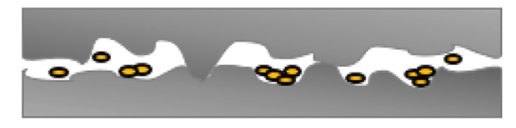

(c)

$20 \mathrm{wt} \%$ oil $+\alpha$-amylase

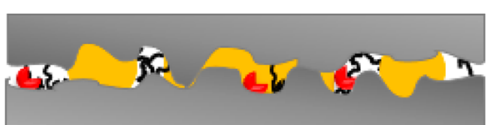

O Emulsion droplet

$\alpha$-amylase

OSA starch chains

Scheme 2. Schematic Representation of Native Starch Particles in the Boundary Regime of a Tribometer at Low (a) and High (b) Starch Concentration in the Absence or Presence of $\alpha$-Amylase (c); Schematic Representation of Starch Based Emulsion Microgel Particles at Low (d) or High (e) Particle Volume Fraction without or with $\alpha$-Amylase (f)
Under
tribological
Under tribological shear $+\alpha$-amylase

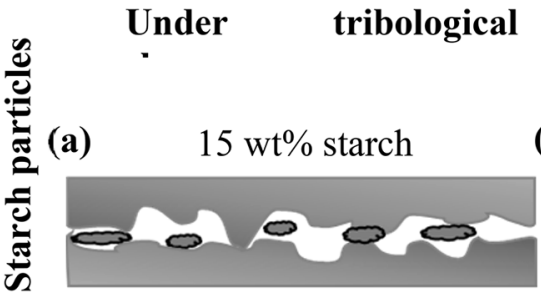

(a) $15 \mathrm{wt} \%$ starch

(b) $20 \mathrm{wt} \% \mathrm{starch}$

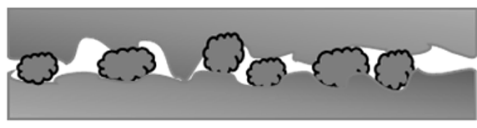

(c)

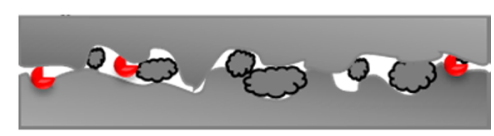

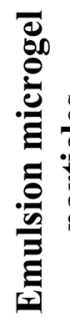

(d)

$60 \mathrm{vol} \%$ particles

(e) $30 \mathrm{vol} \%$ particles

(f)

30 vol\% particles

\section{递

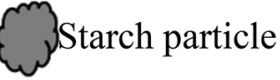 \\ $\alpha$-amylase}
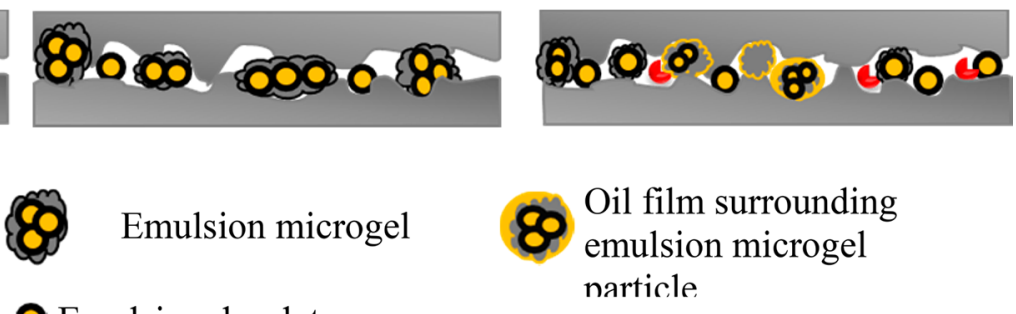

Emulsion microgel

O Emulsion droplet
Oil film surrounding emulsion microgel narticle
(Figure 3b). By aggregating the emulsion droplets would then be able to support the load and reduce the friction coefficient. Scheme $1 \mathrm{a}, \mathrm{b}$ attempts to illustrate the lubrication mechanism of the emulsion droplets at $\bar{U}=3 \mathrm{~mm} \mathrm{~s}^{-1}$. It should be noted that the hydrodynamic regime obtained with the diluted emulsion, resembling sunflower oil alone (Figure S2), might be explained by the coalescence of these aggregating emulsion droplets sliding inside the contact zone at high entrainment speed.

The presence of $\alpha$-amylase dramatically changed the lubrication properties of the emulsion, although its bulk viscosity remained similar to the diluted emulsion without enzyme. As suggested earlier, the OSA starch might have been hydrolyzed and detached from the oil droplet surfaces, leading to their coalescence and the formation of an oil film (Scheme 1c) where the oil dominates the friction. It has also been suggested that surfactant molecules can absorb onto the PDMS surfaces preventing contact between the ball and disc at low entrainment speeds. ${ }^{43}$

Since OSA starch is amphiphilic, some surface active fragments might desorb from the oil droplets and adsorb to the ball and disc, separating the surfaces further compared to sunflower oil, in accordance with previous studies. $23,34,46$

In comparison, starch microgel particles had a significantly larger $(p<0.05)$ friction coefficient values than the emulsion, depending on both the concentration of starch (i.e., 15 or 20 wt \%) used to form the particles and the volume fraction of particles (i.e., $60 \mathrm{vol} \%$ or $30 \mathrm{vol} \%$ ) in solution. Since the friction coefficient of 15 and 20 wt \% starch particles + buffer had similar friction values, it can be assumed that $30 \mathrm{vol} \%$ of particles had very limited lubrication properties in the boundary region, probably due to the low viscosity. At higher volume fraction, i.e., $60 \mathrm{vol} \%$ particles, the concentration of starch influenced the lubrication behavior of the particles. At 15 wt \% starch, only $29 \%$ of the load is predicted to be supported by the starch particles, whereas at $20 \mathrm{wt} \%$ starch, $71 \%$ of the load should be supported by the particles (Table 1 ). The relative indentation of the starch particles being larger than 1 suggests that on entering the contact zone both particle types would be deformed and destroyed. The light scattering results suggest that the particles formed from $15 \mathrm{wt} \%$ starch were broken down more easily during tribological shear compared to the $20 \mathrm{wt} \%$ starch particles. Their differences in lubrication might therefore be explained by their differences in viscosity, so that the viscosity of the $20 \mathrm{wt} \%$ starch particles dispersion lead to a higher drag force, allowing the particles to be entrained and deformed, decreasing the friction via load bearing (Scheme 2b).

Likewise the addition of $\alpha$-amylase to the starch particles decreased the friction coefficient of both starch particles (15 and $20 \mathrm{wt} \%$ ) due to the limited hydrolysis of the starch microgel particles (Scheme 2c), under the action of $\alpha$-amylase 
the particles might lose their structure and be entrained more easily at low speed.

The starch microgel particles with encapsulated oil droplets (Scheme $2 \mathrm{~d}, \mathrm{e}$ ) gave a similar decrease of friction, compared to the emulsion, at both volume fractions ( 60 and 30 vol \%). The oil droplets acted as active fillers and strengthened the emulsion gel so that the emulsion microgel particles were more rigid (see comment in Section 3.4). ${ }^{18}$ At $\bar{U}=3 \mathrm{~mm} \mathrm{~s}^{-1}$, the emulsion microgel particles before and after dilution gave a similar friction coefficient to the emulsion droplets, which implied that $86 \%$ of the load was supported by the emulsion microgel particles. However, regarding the relative indentation, this was estimated at 12.7 and the drag force was estimated high enough to entrain the emulsion microgel particles in the contact zone (Table 1). Similarly to the 20 wt \% starch particles, on entering the contact zone the emulsion microgel particles should therefore become highly compressed and deformed, probably allowing some emulsion droplets to be released in the continuous phase. However, due to the emulsion droplets acting as active filler it is possible that on entering the contact zone the microgel particles would compress until the modulus of the microgel particles approaches the modulus of the encapsulated emulsion droplets allowing the system to support the load rather than completely breaking down. This mechanism would also explain the smaller shift in particle size after tribological shear, compared to the larger shift in particle size observed for the unfilled microgel particles (Figure 6a).

When $\alpha$-amylase was added to the emulsion microgel particles, it is proposed that starch microgel particle fragments are produced as well as emulsion microgel particles surrounded by an oil film (Scheme $2 \mathrm{f}$ ), due to the small amount of emulsion breakdown. The OSA starch adsorbed at the surface of the oil droplets largely remains intact, and the encapsulated oil droplets are able to diffuse into the continuous phase, where they are mostly stable. The released oil droplets thus enhance the lubrication of the filled microgel particles via the dual action of the few oil droplets released and the smaller sized emulsion microgel particles, mimicking the lubrication of the emulsion in the mixed region.

\section{CONCLUSIONS}

Emulsion microgel particles with stimuli-responsiveness to physiological enzyme and shear were proposed and have been realized creating a novel biolubricant additive. In response to tribological shear and enzyme activity, some oil droplets entrapped in the starch microgel particles are released and improved lubrication. Based on confocal fluorescence microscopy, these oil droplets are still stabilized by an adhering OSA starch layer and residual microgel material. Thus, the encapsulation of the oil droplets into these model emulsion microgel particles protected them throughout the biological processing so that their potential lubrication properties were not lost. We expect that this persistently stable model particle can be modified, through the use of other biopolymers, for further applications such as to target and control the delivery of lipophilic nutrients or pharmaceuticals. This shear and enzyme-responsive emulsion microgel particle might reduce the degradation of lipophilic compounds in the presence of harsh physiological environment (i.e., biological shear such as rubbing skin, mastication or gastric motility, biological enzymes, acidic conditions). For instance, the starch microgel particle should further protect the lipophilic bioactive during gastric conditions while degrade in the small intestine. Where further contact with $\alpha$-amylase will allow the destabilization of the oil droplets for increased bioaccessibility of oil-soluble bioactive compounds. If such emulsion microgel particles can be developed, it may be possible to formulate bio responsive particle that offer both lubrication and bioavailability advantages in both industrial and biological applications.

\section{ASSOCIATED CONTENT}

\section{S Supporting Information}

The Supporting Information is available free of charge on the ACS Publications website at DOI: 10.1021/acsami.8b07883.

Flow curves of the emulsion microgel particles produced at $20 \mathrm{wt} \%$ starch (Figure S1); coefficient of friction as a function of entrainment speed at $\bar{U}>10 \mathrm{~mm} \mathrm{~s}^{-1}$ for both the emulsion and the particles (Figures S2 and S3); particle size distributions of the emulsion microgel particles produced at 20 wt \% starch under different physiological conditions (Figure S9); statistical analysis of the friction coefficient values at 3 and $50 \mathrm{~mm} \mathrm{~s}^{-1}$ of the different samples under different conditions (Tables S3 and S5-S8); and theoretical calculations of both the relative indentation and drag force (section S10) (PDF)

\section{AUTHOR INFORMATION}

\section{Corresponding Author}

*E-mail: A.Sarkar@leeds.ac.uk.

ORCID $\odot$

Anwesha Sarkar: 0000-0003-1742-2122

\section{Author Contributions}

The manuscript was written through contributions of all authors. All authors have given approval to the final version of the manuscript.

\section{Funding}

This project has received funding from the European Research Council (ERC) under the European Union's Horizon 2020 research and innovation program (Grant Agreement No. 757993).

\section{Notes}

The authors declare no competing financial interest.

\section{ACKNOWLEDGMENTS}

The authors would like to thank Ingredion for providing the commercial OSA starch and Martin Fuller (Faculty of Biological Science, University of Leeds) for his technical support in electron microscopy.

\section{REFERENCES}

(1) Liu, G.; Liu, Z.; Li, N.; Wang, X.; Zhou, F.; Liu, W. Hairy Polyelectrolyte Brushes-Grafted Thermosensitive Microgels as Artificial Synovial Fluid for Simultaneous Biomimetic Lubrication and Arthritis Treatment. ACS Appl. Mater. Interfaces 2014, 6 (22), 20452-20463.

(2) Sterner, O.; Karageorgaki, C.; Zürcher, M.; Zürcher, S.; Scales, C. W.; Fadli, Z.; Spencer, N. D.; Tosatti, S. G. P. Reducing Friction in the Eye: A Comparative Study of Lubrication by Surface-Anchored Synthetic and Natural Ocular Mucin Analogues. ACS Appl. Mater. Interfaces 2017, 9 (23), 20150-20160.

(3) Timm, K.; Myant, C.; Spikes, H. A.; Grunze, M. Particulate Lubricants in Cosmetic Applications. Tribol. Int. 2011, 44 (12), $1695-1703$

(4) Røn, T.; Chronakis, I.; Lee, S. Polymer 2014, 55, 5708-5717. 
(5) Røn, T.; Patil, N. J.; Ajalloueian, F.; Rishikesan, S.; Zappone, B.; Chronakis, I. S.; Lee, S. Gastric Mucus and Mucuslike Hydrogels: Thin Film Lubricating Properties at Soft Interfaces. Biointerphases 2017, 12 (5), 051001.

(6) Malone, M. E.; Appelqvist, I. A. M.; Norton, I. T. Oral Behaviour of Food Hydrocolloids and Emulsions. Part 1. Lubrication and Deposition Considerations. Food Hydrocolloids 2003, 17 (6), 763773.

(7) Sarkar, A.; Kanti, F.; Gulotta, A.; Murray, B. S.; Zhang, S. Aqueous Lubrication, Structure and Rheological Properties of Whey Protein Microgel Particles. Langmuir 2017, 33, 14699.

(8) Dou, X.; Koltonow, A. R.; He, X.; Jang, H. D.; Wang, Q.; Chung, Y.-W.; Huang, J. Self-Dispersed Crumpled Graphene Balls in Oil for Friction and Wear Reduction. Proc. Natl. Acad. Sci. U. S. A. 2016, 113 (6), 1528-1533.

(9) Garrec, D. A.; Norton, I. T. Kappa Carrageenan Fluid Gel Material Properties. Food Hydrocolloids 2013, 33 (1), 160-167.

(10) Secret, E.; Kelly, S. J.; Crannell, K. E.; Andrew, J. S. EnzymeResponsive Hydrogel Microparticles for Pulmonary Drug Delivery. ACS Appl. Mater. Interfaces 2014, 6 (13), 10313-10321.

(11) Batchelor, H.; Venables, R.; Marriott, J.; Mills, T. The Application of Tribology in Assessing Texture Perception of Oral Liquid Medicines. Int. J. Pharm. 2015, 479 (2), 277-281.

(12) Stokes, J. R.; Boehm, M. W.; Baier, S. K. Oral Processing, Texture and Mouthfeel: From Rheology to Tribology and Beyond. Curr. Opin. Colloid Interface Sci. 2013, 18 (4), 349-359.

(13) Joyce, P.; Whitby, C. P.; Prestidge, C. A. Bioactive Hybrid Particles from Poly(D,L-Lactide-Co-Glycolide) Nanoparticle Stabilized Lipid Droplets. ACS Appl. Mater. Interfaces 2015, 7 (31), 17460-17470.

(14) Porter, C. J. H.; Pouton, C. W.; Cuine, J. F.; Charman, W. N. Enhancing Intestinal Drug Solubilisation Using Lipid-Based Delivery Systems. Adv. Drug Delivery Rev. 2008, 60 (6), 673-691.

(15) Simovic, S.; Barnes, T. J.; Tan, A.; Prestidge, C. A. Assembling Nanoparticle Coatings to Improve the Drug Delivery Performance of Lipid Based Colloids. Nanoscale 2012, 4 (4), 1220-1230.

(16) Torres, O.; Murray, B.; Sarkar, A. Emulsion Microgel Particles: Novel Encapsulation Strategy for Lipophilic Molecules. Trends Food Sci. Technol. 2016, 55 (Supplement C), 98-108.

(17) Torres, O.; Murray, B.; Sarkar, A. Design of Novel Emulsion Microgel Particles of Tuneable Size. Food Hydrocolloids 2017, 71 (Supplement C), 47-59.

(18) Torres, O.; Tena, N. M.; Murray, B.; Sarkar, A. Novel Starch Based Emulsion Gels and Emulsion Microgel Particles: Design, Structure and Rheology. Carbohydr. Polym. 2017, 178, 86-94.

(19) Hamilton, I. E.; Norton, I. T. Modification to the Lubrication Properties of Xanthan Gum Fluid Gels as a Result of Sunflower Oil and Triglyceride Stabilised Water in Oil Emulsion Addition. Food Hydrocolloids 2016, 55, 220-227.

(20) de Wijk, R. A.; Terpstra, M. E. J.; Janssen, A. M.; Prinz, J. F. Perceived Creaminess of Semi-Solid Foods. Trends Food Sci. Technol. 2006, 17 (8), 412-422.

(21) Weenen, H.; Jellema, R. H.; de Wijk, R. A. Sensory SubAttributes of Creamy Mouthfeel in Commercial Mayonnaises, Custard Desserts and Sauces. Food Quality and Preference 2005, 16 (2), 163-170.

(22) Ravanfar, R.; Celli, G. B.; Abbaspourrad, A. Controlling the Release from Enzyme-Responsive Microcapsules with a Smart Natural Shell. ACS Appl. Mater. Interfaces 2018, 10 (6), 6046-6053.

(23) Xu, Y.; Zheng, Y.; Wu, L.; Zhu, X.; Zhang, Z.; Huang, Y. Novel Solid Lipid Nanoparticle with Endosomal Escape Function for Oral Delivery of Insulin. ACS Appl. Mater. Interfaces 2018, 10 (11), 93159324.

(24) Xia, F.; Fan, W.; Jiang, S.; Ma, Y.; Lu, Y.; Qi, J.; Ahmad, E.; Dong, X.; Zhao, W.; Wu, W. Size-Dependent Translocation of Nanoemulsions Via Oral Delivery. ACS Appl. Mater. Interfaces 2017, 9 (26), 21660-21672.

(25) Sun, L.; Wang, Y.; Jiang, T.; Zheng, X.; Zhang, J.; Sun, J.; Sun, C.; Wang, S. Novel Chitosan-Functionalized Spherical Nanosilica
Matrix as an Oral Sustained Drug Delivery System for Poorly WaterSoluble Drug Carvedilol. ACS Appl. Mater. Interfaces 2013, 5 (1), 103-113.

(26) Nguyen, C. T. H.; Webb, R. I.; Lambert, L. K.; Strounina, E.; Lee, E. C.; Parat, M.-O.; McGuckin, M. A.; Popat, A.; Cabot, P. J.; Ross, B. P. Bifunctional Succinylated E-Polylysine-Coated Mesoporous Silica Nanoparticles for Ph-Responsive and Intracellular Drug Delivery Targeting the Colon. ACS Appl. Mater. Interfaces 2017, 9 (11), 9470-9483.

(27) Minekus, M.; Alminger, M.; Alvito, P.; Ballance, S.; Bohn, T.; Bourlieu, C.; Carriere, F.; Boutrou, R.; Corredig, M.; Dupont, D.; Dufour, C.; Egger, L.; Golding, M.; Karakaya, S.; Kirkhus, B.; Le Feunteun, S.; Lesmes, U.; Macierzanka, A.; Mackie, A.; Marze, S.; McClements, D. J.; Menard, O.; Recio, I.; Santos, C. N.; Singh, R. P.; Vegarud, G. E.; Wickham, M. S. J.; Weitschies, W.; Brodkorb, A. A Standardised Static in Vitro Digestion Method Suitable for Food - an International Consensus. Food Funct. 2014, 5 (6), 1113-1124.

(28) Laguna, L.; Farrell, G.; Bryant, M.; Morina, A.; Sarkar, A. Relating Rheology and Tribology of Commercial Dairy Colloids to Sensory Perception. Food Funct. 2017, 8 (2), 563-573.

(29) Sweedman, M. C.; Schäfer, C.; Gilbert, R. G. Aggregate and Emulsion Properties of Enzymatically-Modified Octenylsuccinylated Waxy Starches. Carbohydr. Polym. 2014, 111, 918-927.

(30) Tesch, S.; Gerhards, C.; Schubert, H. Stabilization of Emulsions by Osa Starches. J. Food Eng. 2002, 54 (2), 167-174.

(31) Anvari, M.; Joyner, H. S. Effect of Formulation on StructureFunction Relationships of Concentrated Emulsions: Rheological, Tribological, and Microstructural Characterization. Food Hydrocolloids 2017, 72 (Supplement C), 11-26.

(32) Dickinson, E. On the Road to Understanding and Control of Creaminess Perception in Food Colloids. Food Hydrocolloids 2018, 77, 372.

(33) de Wijk, R. A.; Prinz, J. F.; Engelen, L.; Weenen, H. The Role of A-Amylase in the Perception of Oral Texture and Flavour in Custards. Physiol. Behav. 2004, 83 (1), 81-91.

(34) Dresselhuis, D. M.; de Hoog, E. H. A.; Cohen Stuart, M. A.; Vingerhoeds, M. H.; van Aken, G. A. The Occurrence of in-Mouth Coalescence of Emulsion Droplets in Relation to Perception of Fat. Food Hydrocolloids 2008, 22 (6), 1170-1183.

(35) Adams, S.; Frith, W. J.; Stokes, J. R. Influence of Particle Modulus on the Rheological Properties of Agar Microgel Suspensions. J. Rheol. 2004, 48 (6), 1195-1213.

(36) Ching, S. H.; Bansal, N.; Bhandari, B. Rheology of EmulsionFilled Alginate Microgel Suspensions. Food Res. Int. 2016, 80, 50-60.

(37) Mueller, S.; Llewellin, E. W.; Mader, H. M. The Rheology of Suspensions of Solid Particles. Proc. R. Soc. London, Ser. A 2010, 466, 1201.

(38) Alazemi, A. A.; Etacheri, V.; Dysart, A. D.; Stacke, L.-E.; Pol, V. G.; Sadeghi, F. Ultrasmooth Submicrometer Carbon Spheres as Lubricant Additives for Friction and Wear Reduction. ACS Appl. Mater. Interfaces 2015, 7 (9), 5514-5521.

(39) de Vicente, J.; Spikes, H. A.; Stokes, J. R. Viscosity Ratio Effect in the Emulsion Lubrication of Soft Ehl Contact. J. Tribol. 2006, 128 (4), 795-800.

(40) Dedinaite, A.; Pettersson, T.; Mohanty, B.; Claesson, P. M. Lubrication by Organized Soft Matter. Soft Matter 2010, 6 (7), 15201526.

(41) Chojnicka, A.; de Jong, S.; de Kruif, C. G.; Visschers, R. W. Lubrication Properties of Protein Aggregate Dispersions in a Soft Contact. J. Agric. Food Chem. 2008, 56 (4), 1274-1282.

(42) Fernández Farrés, I.; Norton, I. T. The Influence of Co-Solutes on Tribology of Agar Fluid Gels. Food Hydrocolloids 2015, 45 (Supplement C), 186-195.

(43) Liu, K.; Tian, Y.; Stieger, M.; van der Linden, E.; van de Velde, F. Evidence for Ball-Bearing Mechanism of Microparticulated Whey Protein as Fat Replacer in Liquid and Semi-Solid Multi-Component Model Foods. Food Hydrocolloids 2016, 52 (Supplement C), 403414. 
(44) de Vicente, J.; Stokes, J. R.; Spikes, H. A. Lubrication Properties of Non-Adsorbing Polymer Solutions in Soft Elastohydrodynamic (Ehd) Contacts. Tribol. Int. 2005, 38 (5), 515-526.

(45) Tatara, Y. On Compression of Rubber Elastic Sphere over a Large Range of Displacements-Part 1: Theoretical Study. J. Eng. Mater. Technol. 1991, 113 (3), 285-291.

(46) Pradal, C.; Stokes, J. R. Oral Tribology: Bridging the Gap between Physical Measurements and Sensory Experience. Current Opinion in Food Science 2016, 9 (SupplementC), 34-41. 